\title{
A PROBLEM ON A GEOMETRIC PROPERTY OF LEMNISCATES
}

\author{
J. S. HWANG
}

\begin{abstract}
Let $R^{3}$ be the Eulcidean space and let $p_{n}$ be the product defined by $p_{n}\left(W, W_{k}\right)=\mathbb{I}_{k=1}^{n}\left|W-W_{k}\right|, W, W_{k} \in R^{3}$, where $\left|W-W_{k}\right|$ is the distance between $W$ and $W_{k}$. Let $C(n)$ be the class of all such products with the same degree $n$. For any product $p$, we call $E(p)=\{W: p(W)<1\}$ the lemniscate of $p$. We recently proved that if $p_{n}\left(W, W_{k}\right)$ and $p_{n}^{*}\left(W, W_{k}^{*}\right)$ are two products in $C(n)$ such that $E\left(p_{n}\right) \subseteq E\left(p_{n}^{*}\right)$, and if all zeros $W_{k}$ of $p_{n}$ lie on the same plane, then $p_{n} \equiv p_{n}^{*}$. We then asked whether this result is sharp. In this note, we answer this question in the affirmative sense.
\end{abstract}

Let $W=\left(w_{1}, w_{2}, w_{3}\right)$ and $W_{k}=\left(w_{k 1}, w_{k 2}, w_{k 3}\right)$ be points in the Euclidean space $R^{3}$ and let $\left|W-W_{k}\right|$ be the distance between $W$ and $W_{k}$. Denote by $C(n)$ the class of all products $p_{n}$ of the same degree $n$ defined by

$$
p_{n}\left(W, W_{k}\right)=\prod_{k=1}^{n}\left|W-W_{k}\right|, \quad W, W_{k} \in R^{3}
$$

For any product $p$, we call $E(p)=\{W: p(W) \leqslant 1\}$ the lemniscate of $p$. Recently in [1], we proved the following

TheORem 1. Let $p_{n}\left(W, W_{k}\right)$ and $p_{n}^{*}\left(W, W_{k}^{*}\right)$ be two products in $C(n)$ such that $E\left(p_{n}\right) \subseteq E\left(p_{n}^{*}\right)$. If all zeros $W_{k}$ of $p_{n}$ lie on the same plane, then $p_{n} \equiv p_{n}^{*}$.

This theorem describes an important property of lemniscates of products in $C(n)$. For any $p$ in $C(n)$, we say that its lemniscate $E(p)$ is extremal if it cannot be contained in any other lemniscate $E\left(p^{*}\right), p^{*} \in C(n)$. With this term, the above theorem says that if all zeros of a product $p$ lie on the same plane then $E(p)$ is extremal. As was remarked in [1, p. 347], this theorem can be extended from $R^{3}$ to $R^{n}$. However, problems of this kind essentially need to be considered in $R^{3}$.

On the other hand, if all zeros of $p$ with two exceptions lie on the same plane then the theorem is no longer true; see our example in [1]. We then ask whether this theorem is still true if all zeros of $p$ with one exception lie on the same plane. In other words, whether this theorem is sharp with regard to the number of exceptional points. In this note, we shall answer this question in the affirmative sense by the following construction.

Received by the editors August 7, 1980.

AMS (MOS) subject classifications (1970). Primary 30A21, 26A75; Secondary 30A06, 30 A42.

Key words and phrases. Product, lemniscate, extremal property.

(c) 1981 American Mathematical Society 0002-9939/81/0000-0316/\$01.75 
THeOREM 2. Let $0<a<1$ be fixed, $\varepsilon>0$ sufficiently small, and

$$
\begin{aligned}
p_{n+1}^{2}\left(W, a, \varepsilon e^{i 2 k \pi / n}\right)= & {\left[w_{1}^{2}+w_{2}^{2}+\left(w_{3}-a\right)^{2}\right] } \\
& \cdot \prod_{k=1}^{n}\left[\left(w_{1}-\varepsilon \cos \frac{2 k \pi}{n}\right)^{2}+\left(w_{2}-\varepsilon \sin \frac{2 k \pi}{n}\right)^{2}+w_{3}^{2}\right] .
\end{aligned}
$$

If $n \varepsilon^{2}$ is large, but $n \varepsilon^{3}$ is small, then $E\left(p_{n+1}\right) \subset E\left(p_{n+1}^{0}\right)$, where $p_{n+1}^{0}(W)=\Pi_{k=1}^{n+1}|W|$ and $E\left(p_{n+1}^{0}\right)=\{W:|W| \leqslant 1\}$.

Proof. Let $S=\{W:|W|=1\}$. Then it is sufficient to prove that

$$
p_{n+1}\left(W, a, e^{i 2 k \pi / n}\right)>1 \text { for any } W \in S .
$$

For convenience, we shall use polar coordinates and we write $f(\theta, \phi)$ to denote $p_{n+1}^{2}$ in polar form, with respect to an arbitrary point $p(\cos \theta \cos \phi, \sin \theta \cos \phi$, $\sin \phi)$ on $S$, where $0 \leqslant \theta<2 \pi$ and $-\pi / 2 \leqslant \phi \leqslant \pi / 2$. Clearly we have

$$
f(\theta, \phi)=\left(1+a^{2}-2 a \sin \phi\right) \prod_{k=1}^{n}\left\{1+\left[\varepsilon^{2}-2 \varepsilon \cos \phi \cos \left(\theta-\frac{2 k \pi}{n}\right)\right]\right\} .
$$

By taking the logarithm on both sides of (2) and observing the basic equality $\log (1+x)=x-x^{2} / 2+O\left(x^{3}\right)$, where $x$ is small, we obtain

$$
\begin{aligned}
\log f(\theta, \phi)= & \log \left(1+a^{2}-2 a \sin \phi\right)+n \varepsilon^{2}-2 \varepsilon \cos \phi \sum_{k=1}^{n} \cos \left(\theta-\frac{2 k \pi}{n}\right) \\
& -2 \varepsilon^{2} \cos ^{2} \phi \sum_{k=1}^{n} \cos ^{2}\left(\theta-\frac{2 k \pi}{n}\right)+n O\left(\varepsilon^{3}\right) .
\end{aligned}
$$

By using the basic formulas of trigonometric sums, we have

$$
\sum_{k=1}^{n} \cos \frac{2 j k \pi}{n}=0, \quad \sum_{k=1}^{n} \sin \frac{2 j k \pi}{n}=0, \quad \text { for } j=1,2 .
$$

This yields

$$
\sum_{k=1}^{n} \cos \left(\theta-\frac{2 k \pi}{n}\right)=0 \text { and } \sum_{k=1}^{n} \cos ^{2}\left(\theta-\frac{2 k \pi}{n}\right)=\frac{n}{2} .
$$

By substituting (4) into (3), we get

$$
\log f(\theta, \phi)=\log \left(1+a^{2}-2 a \sin \phi\right)+n \varepsilon^{2} \sin ^{2} \phi+n O\left(\varepsilon^{3}\right) .
$$

The number $n O\left(\varepsilon^{3}\right)$, by its very meaning must tend to zero, as $\varepsilon \rightarrow 0$. Clearly, if $-\pi / 2 \leqslant \phi \leqslant 0$, then (1) follows from (5), i.e.

$$
\log f(\theta, \phi) \geqslant \log \left(1+a^{2}\right)+n O\left(\varepsilon^{3}\right)>0, \quad \text { as } \varepsilon \rightarrow 0 .
$$

On the other hand, if $0<\phi \leqslant \pi / 2$, we consider two cases: either $\sin \phi<a / 3$ or $\sin \phi>a / 3$. The first case gives

$$
a^{2}-2 a \sin \phi>a^{2}-2 a^{2} / 3=a^{2} / 3,
$$

so that

$$
\log f(\theta, \phi) \geqslant \log \left(1+a^{2} / 3\right)+n O\left(\varepsilon^{3}\right)>0, \quad \text { as } \varepsilon \rightarrow 0
$$


And the last case yields

$$
\log f(\theta, \phi)>\log (1-a)^{2}+n \varepsilon^{2} a^{2} / 9+n O\left(\varepsilon^{3}\right)>0, \quad \text { as } \varepsilon \rightarrow 0
$$

provided

$$
n \geqslant\left[9(1+2 \log (1 /(1-a))) /\left(\varepsilon^{2} a^{2}\right)\right] \text {. }
$$

Thus by first choosing $\varepsilon>0$ sufficiently small and then choosing $n$ such that $n \varepsilon^{2}$ is large, but $n \varepsilon^{3}$ is small, we conclude that $\log f(\theta, \phi)>0$. This yields (1) and the proof is complete.

\section{REFERENCES}

1. P. Erdös and J. S. Hwang, On a geometric property of lemniscates, Aequationes Math. 17 (1978), 344-347.

Institute of Mathematics, Academia Sinica, Taipei, Tatwan 